\title{
MAPPING THE SOLAR CORONA IN X-RAY LINES OF OVII AND NeIX
}

\author{
R. C. CATURA, L. W. ACTON, and A. J. MEYEROTT \\ Lockheed Palo Alto Research Laboratory Palo Alto, Calif. 94304, U.S.A. \\ and \\ J. L. CULHANE \\ Mullard Space Science Laboratory, University College London
}

Some preliminary results of a rocket experiment, which utilized collimated Bragg Crystal spectrometers to map solar X-ray line emissions from He-like ions of oxygen and neon are presented. The two spectrometers used KAP crystals with a common field of view $1.7^{\prime}$ FWHM in the narrow dimension. The spectrometers were scanned

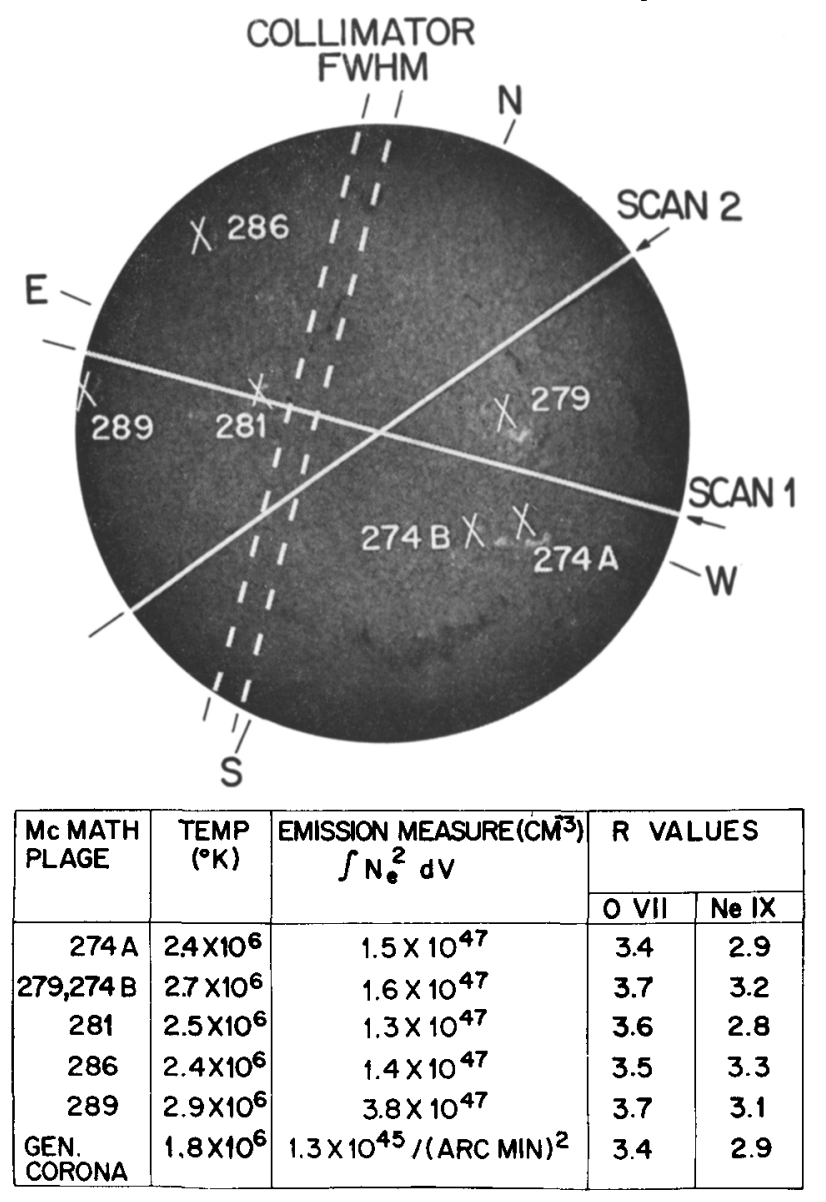

Fig. 1. Location of the emitting regions as determined from two fan beam scans of the disk are shown by crosses on a $\mathrm{H} \alpha$ photograph of the Sun. The scan paths, the relative size of the collimator field of view, and parameters evaluated by this experiment are also shown. 
in wavelength once each second to observe the resonance, intersystem and forbidden lines of Ovil and NeIX.

The spatial distribution of these line emissions was measured on April 29, 1971 near $1630 \mathrm{UT}$ and has been resolved into contributions from at least six regions of activity on the sun. By utilizing intensities of the O VII and Ne IX resonance lines and assuming an isothermal plasma we have calculated the temperature of each region and the corresponding value of emission measure $\left(\int N_{\mathrm{e}}^{2} \mathrm{~d} v\right)$. The parameter $R$, the ratio of forbidden $\left({ }^{3} S_{1}-{ }^{1} S_{0}\right)$ to intersystem $\left({ }^{3} P-{ }^{1} S_{0}\right)$ line intensities, has also been calculated.

These data are shown in Figure 1. Values for the general corona were obtained when the field of view was free of plage areas and represent averaging over a $1.7^{\prime}$ wide strip extending approximately from pole to pole. Values of KAP crystal reflectively have been obtained from the measurements of Liefeld et al. (1970). The abundances of oxygen and neon used for these calculations were provided by Drs Dupree and Withbroe and are coronal values derived from XUV data obtained by the Harvard experiment on OSO 4.

No significant variation of the value $R$ is found from feature to feature on the disk. According to a recent paper by Freeman et al. (1971), this places an upper limit of $6 \times 10^{9} \mathrm{~cm}^{-3}$ on the electron density in the coronal regions observed in the experiment.

\section{Acknowledgements}

This work was supported by the National Aeronautics and Space Administration under Contract NASw-1834 and by the Lockheed Independent Research Program.

\section{References}

Freeman, F. F., Gabriel, A. H., Jones, B. B., and Jordan, C.: 1971, Phil. Trans. Roy. Soc. London A270, 127.

Liefeld et al.: 1970, Adv. X-Ray Analysis 13, 378.

\section{DISCUSSION}

A. B. C. Walker: Can you obtain an integrated emission measure for the entire disk by summing your data?

R. C. Catura: We have not yet analysed some low intensity emission from several plages near the west limb. When this analysis is completed we can obtain the emission measure for the entire disk.

$S$. R. Pottasch: To get good agreement for $N_{\mathrm{e}}^{2} V$ from the oxygen and neon lines you only need the correct $\mathrm{O}: \mathrm{Ne}$ ratio and not the absolute values with respect to hydrogen. What value of the $\mathrm{O}: \mathrm{Ne}$ ratio did you use?

R. C. Catura: In this analysis we have used coronal values of $\mathrm{O}$ and $\mathrm{Ne}$ abundance which were obtained by Dr Dupree of Harvard College Observatory. These values give an O: Ne ratio of 20. 